\title{
A Mechanism for Hydrogen Embrittlement in Martensitic Steel Based on Hydrogen Dilation
}

\author{
Michael F. McGuire ${ }^{1, *}$ and Roy J. Matway ${ }^{2}$ \\ 1 McGuire Associates, 2901 Smallman St., Pittsburgh PA, USA 15201 \\ 2 Carnegie Mellon Materials Science and Engineering, 5000 Forbes Ave., Pittsburgh PA USA 15213; \\ matway@cmu.edu \\ * Correspondence author: mfm304@gmail.com
}

\begin{abstract}
Martensitic steels are used at a wide range of strength levels in environments which expose them to hydrogen or water vapor over a large range of partial pressures and temperatures. Hydrogen can cause catastrophic failures under many seemingly benign conditions. The effect of hydrogen on the dimension stability of high strength martensitic steels under such conditions has been poorly understood, and existing models do not seem to adequately account for it. Experiments were conducted to measure the variation in volume due to the uptake of hydrogen of such steels under near-ambient conditions, and the results were compared to theoretical estimates derived from the density of defects acting as hydrogen traps. Based on these results a new model for hydrogen embrittlement was developed. The hydrogen lattice dilation (HLD) model isolates volume expansion as a primary driver of hydrogen embrittlement. It provides and distinguishes two modes of failure acceleration: the fast, brittle, stress-intensity independent cracking under higher static crack loading, and a slower, highly stress-intensity dependent tearing mode at lower stress intensity. The relationship between the two is explained, as is how hydrogen absorption by defects accounts for the crack threshold and crack velocity of each.
\end{abstract}

Keywords: hydrogen embrittlement; martensite; dislocation density; fracture mechanics; stainless steel

\section{Introduction}

Research on hydrogen embrittlement has focused singularly on mapping and identifying mechanisms by which hydrogen degrades the intrinsic mechanical behavior of metals. Indeed, the term hydrogen embrittlement presupposes that the phenomenon must arise from a profound degrading of intrinsic mechanical properties. This assumption of degradation has dominated research on the phenomenon at the expense of other effects of hydrogen. Many compelling mechanisms for hydrogen degradation have been proposed and their existence verified. These have been proposed and reviewed elsewhere [1-9]. But, while many mechanisms are accepted, they have not yet provided the comprehensive quantitative agreement that accompanies an accepted model. So, while degradation of the intrinsic alloy mechanical properties has been the primary focus, less attention has been given to ways hydrogen might alter the stress state itself. Here, the lattice dilation caused by hydrogen is paramount. Lattice expansion causes stress, and any comprehensive failure model must take all stresses into account. However, in the vast literature on hydrogen embrittlement there is little to be found on the dilation that trapped hydrogen causes, let alone the magnitude of the stress-altering effect it might have. It is implicitly assumed to be negligible. However, closer inspection of hydrogen trapping defects, their binding energy and concentration, particularly in deformed metals, makes this assumption tenuous. At least some part of what is believed to be hydrogen degradation may be due to hydrogen altering the stresses at the locus of hydrogen embrittlement by the mechanical effect of dilation. This effect should be readily quantified, which would then permit the separate and distinct degrading mechanisms to 
be more clearly assessed, paving the way for a comprehensive model for hydrogen embrittlement.

Understanding the mechanical effect of hydrogen in steel is not a dismissal of other effects hydrogen has. Dilation effects can coexist with degradation mechanisms which affect intrinsic mechanical behavior. So, by understanding the effect dilation has, we can separate and distinguish the occurrence of the more extensively studied degradation effects.

Hydrogen has a large partial molar volume in steel [10], as do other interstitial solutes such as carbon and nitrogen. However, hydrogen is quite mobile at ambient temperatures while carbon and nitrogen are very immobile. Thus, hydrogen has a unique ability to quickly diffuse even at room temperature to hydrogen traps, which other interstitial elements do not have. It also has diminishingly small solubility in ferrite due to its enthalpy of $\sim 26 \mathrm{~kJ} / \mathrm{mol}$, but it has a great affinity for defects of all types. To a first approximation hydrogen in martensite is only associated with defects. If we categorize defects by the strength with which they bond with hydrogen, we can cite three categories: weak, medium, and strong [11]. In the weak category we place alloy atoms and coherent precipitate interfaces. These defects have binding energies greater (weaker binding) than $-15 \mathrm{~kJ} / \mathrm{mol}$. Dislocations and vacancies are in the medium category, with binding energies between -15 and $-50 \mathrm{~kJ} / \mathrm{mol}$. In the strong category are incoherent interfaces, such as grain boundaries and incoherent precipitates, with binding energies lower (stronger binding) than $-50 \mathrm{~kJ} / \mathrm{mol}$. This wide range of energies means that low-binding energy defects are minimally saturated with hydrogen under ambient conditions, while defects (also known as "traps") in the strong category are nearly totally saturated. Medium-strength traps are partly saturated under some ambient, and sometimes transient conditions, but these traps lose hydrogen rapidly with increasing temperature and decreasing hydrogen activity.

Steels contain many types of defects. However, this study focuses on the effect of hydrogen trapping by dislocations in martensitic steels, without considering trapping by coherent hardening phases incorporated into some ultra-high strength steels. While hydrogen-trap-strengthening coherent precipitates are important to alloy design and performance, they would add an unnecessary analytical complication. Nor has much attention been paid to strong traps, which certainly exist in all steels. This is because their hydrogen content does not vary greatly with stress, deformation, or temperature, nor does their trap density change except by thermal processing. Our focus is on the medium strength defects because their hydrogen saturation level can change with environmental conditions and because their population can be drastically altered by deformation and stress. Dislocation levels are intrinsically high in martensitic steels. Their correlation with strength is well known, and since deformation creates dislocations, they are densely distributed near crack tips. The binding energy of hydrogen to dislocations varies with position around the dislocation core. The hydrogen atoms in the core of either dislocation type are tightly bonded with binding energies variously estimated from -45 to over -55 $\mathrm{kJ} / \mathrm{mol}$. Edge dislocations also bind hydrogen in the strain fields by amounts estimated to be between -20 and $-30 \mathrm{~kJ} / \mathrm{mol}$. If we consider just the core-located hydrogen, saturation will result in one hydrogen atom per atomic plane per dislocation. This is:

$$
[H]=\rho /[F e]
$$

where $[\mathrm{H}]$ is the atomic reaction hydrogen, $\rho$ is the dislocation density, and $[\mathrm{Fe}]$ is the density of matrix iron atoms per atomic plane, taken as $1.8 \times 10^{15} / \mathrm{cm}^{2}$.

Thus, a dislocation density of $10^{15} / \mathrm{m}^{2}$ will imply a saturation level of $1 / 1.8 \times 10^{-4}$ atomic fraction, or $5.5 \times 10^{-5}$ atom fraction hydrogen $(\sim 1 \mathrm{wppm})$. If the four strain field sites of edge dislocations were occupied as well, and edge dislocations were half the dislocation population then there would be an average of three hydrogen atoms per dislocation per atomic plane. Then Eq. 2 would become that representing full saturation: 


$$
[H]=3 \rho /[\mathrm{Fe}]
$$

and the saturation level would be $\sim 3 \mathrm{wppm}$. A dislocation density of $10^{18} / \mathrm{m}^{2}$, according to Nagumo [12], would result in a saturation density of $\sim 3000 \mathrm{wppm}$. We are concerned with lower, normal levels of dislocations. As-quenched martensitic steel typically has a minimum dislocation density of at least $10^{15} / \mathrm{m}^{2}$ [13], which can saturate at $3 \mathrm{wppm}$, as discussed earlier. This dislocation density can increase by one to two orders of magnitude with deformation and higher carbon levels [12,14], increasing potential hydrogen concentration by the same multiple.

Vacancies appear also to play an important role in trapping hydrogen and have a binding energy estimated by Nagumo [15] of $44 \mathrm{~kJ} / \mathrm{mol}$, similar to dislocation cores. Their density by [15] is estimated to reach a concentration of $10^{-4}$ atomic fraction with deformation. This would at saturation add a potential $1.8 \mathrm{wppm}$ of hydrogen for each hydrogen/vacancy pair. Clusters of vacancies could trap more hydrogen, but clusters appear to be unstable and are lost to annealing below $500 \mathrm{~K}$. Vacancies themselves are annihilated at $650 \mathrm{~K}$ [16].

This study does not attempt to determine the exact mechanisms by which deformation produces defects which trap hydrogen. We need only to measure the lattice expansion which accompanies hydrogen absorption both with and without deformation. This is sufficient for our model because it is the lattice dilation itself which is viewed to cause the diminution of fracture toughness, and this is how the model should be judged. Again, we are not attempting to verify or contest any research on the trapping of hydrogen. We do try to understand it, but our objective is to measure lattice expansion caused by hydrogen and determine what effect that has on crack stability as measured by fracture toughness.

We have invoked the simpler tenets of classical mechanics in this study and have used continuum mechanics rather than strain gradient plasticity for two reasons. First, the statistically stored dislocation (SSD) density of our chosen steels is comparable to or greater than the geometrically necessary dislocation (GND) density. Second, the fracture process zone (FPZ) size ranges from $\sim 0.001 \mathrm{~mm}$ to over $0.1 \mathrm{~mm}$, which is within the domain of classical mechanics. We examine the appropriateness of this choice in the Discussion section. Crack stability is the focus, because the full range of hydrogen's effects on steel seem best shown by fracture toughness testing and an abundance of research has been done in this area.

The structure of this manuscript can be thought of as consisting of two parts. In part one, which starts at the beginning of the next section, we show experimentally that hydrogen causes significant lattice expansion, especially in deformed regions, and attempt to account for that expansion based on the research of others. In the second part, which starts at section 4.2, we propose a model which attempts to show how the hydrogen-induced expansion can account for the two manifestations of hydrogen embrittlement, which are seen in both fixed crack mouth opening and slow rising $\mathrm{K}$ fracture toughness tests.

\section{Materials and Methods}

Table 1 shows the composition of the martensitic stainless steels used in this study. X-ray fluorescence spectroscopy was used to obtain the weight percent $(\mathrm{wt} \%)$ of all elements except for C, S, \& N (LECO method). The mechanical properties in Table 2 show estimates for S35500 and S44000 derived from hardness and bending tests, while the G43400 values are from [8]. A modified 4340 used in [8] is included for comparison. A schematic of the major specimen handling steps is shown in Fig. 1. 


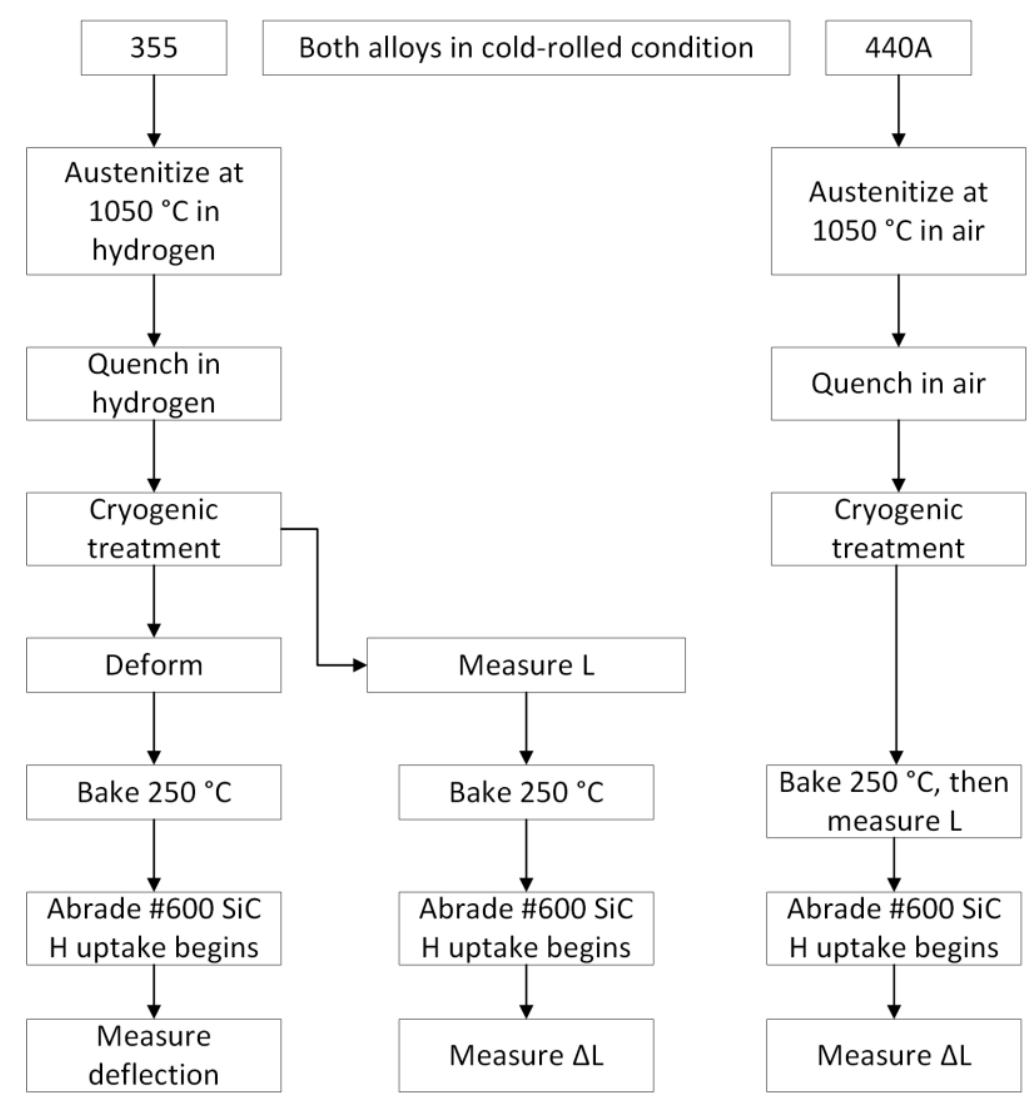

Fig. 1 Schematic of major specimen handling steps.

Specimens were fabricated to allow measurement of the amount of expansion caused by the absorption of hydrogen under various conditions. The 355 specimens were $15 \mathrm{~cm}$ in length by $3 \mathrm{~cm}$ in width and $0.025 \mathrm{~cm}$ in thickness, sheared from cold-rolled and leveled sheet. The 355 specimens were austenitized in a hydrogen atmosphere at $1050{ }^{\circ} \mathrm{C}$ and quenched. The 355 was the principal steel in the study because in the as-quenched condition its yield strength was expected to be ideally $1000 \mathrm{MPa}$, and its thickness permitted easy deformation. In addition to the 355 alloy, parallel tests were conducted on a second alloy, $440 \mathrm{~A}$ to check the results on the primary 355 alloy. The $440 \mathrm{~A}$ specimens were $30 \mathrm{~cm}$ in length by $3 \mathrm{~cm}$ in width and $0.20 \mathrm{~cm}$ in thickness, also sheared from coldrolled and leveled sheet. The 440A specimens were heat treated by austenitizing in air at $1050{ }^{\circ} \mathrm{C}$ and air quenching to room temperature. $440 \mathrm{~A}$ specimens were not pickled to remove oxide. This was done abrasively, purposely leaving a chromium depleted surface layer. The $440 \mathrm{~A}$ alloy was stress relieved at $250{ }^{\circ} \mathrm{C}$ for 180 minutes. All specimens were soaked in liquid nitrogen for 5 minutes to minimize retained austenite. The process was intended to produce a martensitic structure which varied principally in the amount of interstitial carbon. The $1050{ }^{\circ} \mathrm{C}$ austenitizing of $440 \mathrm{~A}$, which according to the manufacturer provides an intentional carbide phase, leaves the martensite with approximately 0.40 to $0.45 \mathrm{wt} \%$ carbon in solution. As-quenched martensite at these carbon levels is reported to have a dislocation density of $\sim \times 10^{11} / \mathrm{cm}^{2}$ and $\sim 15 \times 10^{11} / \mathrm{cm}^{2}$ for 355 and 440A, respectively [13]. These alloys bracket 4340 (SA-372-L) in terms of carbon content, and therefore presumably also in dislocation density when in the martensitic state [13]. They were chosen for this similarity along with their availability as flat-rolled alloys. 

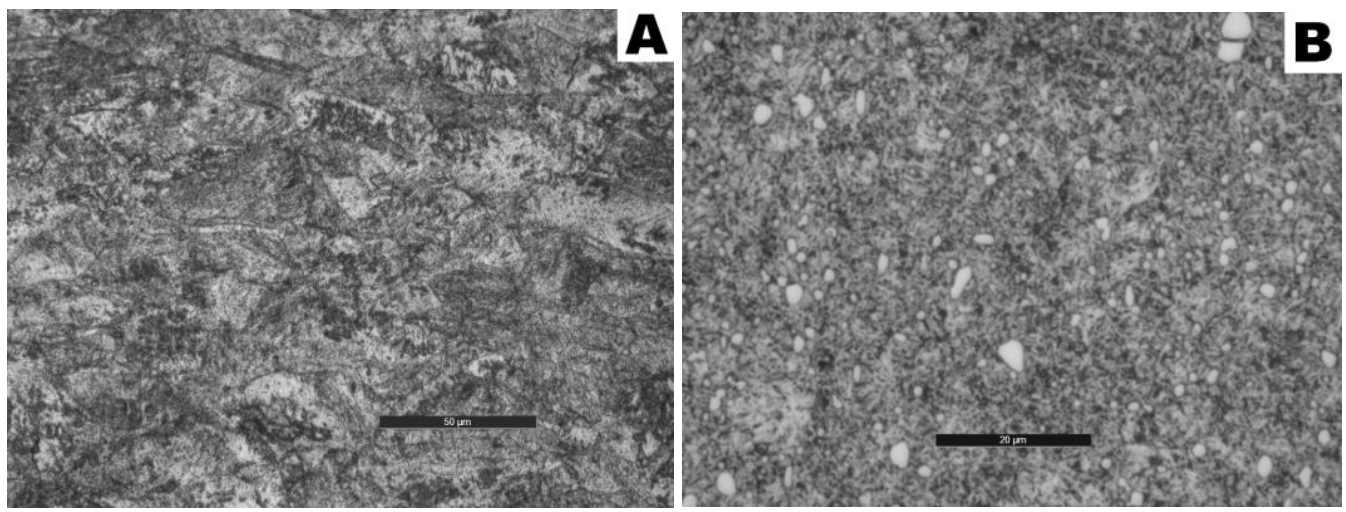

Fig. 2 Microstructures of A) 355, and B) 440A.

The microstructures are shown in Fig. 2. The 355 in Fig. 2A shows the desired fully martensitic structure with an absence of delta ferrite. The 440A in Fig. 2B also shows a fully martensitic structure but with undissolved carbides, and an absence of retained austenite.

Table 1. Chemical compositions in wt $\%$. Data for SA372-L through DOT-3AAX are from [8].

\begin{tabular}{cccccccccccc}
\hline UNS & Grade & $\mathrm{C}$ & $\mathrm{Mn}$ & $\mathrm{P}$ & $\mathrm{S}$ & $\mathrm{Si}$ & $\mathrm{Cr}$ & $\mathrm{Ni}$ & $\mathrm{Mo}$ & $\mathrm{Cu}$ & $\mathrm{N}$ \\
\hline S35500 & 355 & 0.15 & 0.93 & 0.019 & 0.001 & 0.11 & 15.44 & 4.53 & 2.75 & 0.07 & 0.13 \\
\hline S44000 & 440A & 0.61 & 0.51 & 0.020 & 0.001 & 0.39 & 16.70 & 0.33 & 0.04 & 0.11 & 0.04 \\
\hline G43400 & SA372-L & 0.40 & 0.75 & 0.006 & 0.007 & 0.28 & 0.82 & 1.93 & 0.26 & 0.1 & $\mathrm{nr}$ \\
\hline G43400 & DOT-3T & 0.45 & 083 & 0.013 & 0.006 & 0.25 & 0.97 & $\mathrm{nr}$ & 0.18 & $\mathrm{nr}$ & $\mathrm{nr}$ \\
\hline G43400 & SA372-J & 0.47 & 0.92 & 0.012 & 0.003 & 0.30 & 0.96 & $\mathrm{nr}$ & 0.19 & $\mathrm{nr}$ & $\mathrm{nr}$ \\
\hline \multirow{2}{*}{ G43300 } & DOT- & \multirow{2}{*}{0.29} & \multirow{2}{*}{0.62} & \multirow{2}{*}{0.008} & \multirow{2}{*}{0.004} & \multirow{2}{*}{0.28} & \multirow{2}{*}{0.92} & \multirow{2}{*}{$\mathrm{nr}$} & \multirow{2}{*}{0.19} & \multirow{2}{*}{$\mathrm{nr}$} & \multirow{2}{*}{$\mathrm{nr}$} \\
\hline
\end{tabular}

Table 2 Mechanical properties. Data for SA372-L et al are from [8].

\begin{tabular}{cccc}
\hline UNS & Grade & $\begin{array}{c}\text { Yield } \\
\text { strength }\end{array}$ & $\begin{array}{c}\text { Tensile } \\
\text { strength }\end{array}$ \\
\hline & & & \\
\hline S35500 & 355 & 1050 & 1300 \\
\hline S44000 & 440A & 1200 & 1500 \\
\hline G43400 & SA372-L & 1053 & 1300 \\
\hline G43300 & DOT-3T & 900 & 1001 \\
\hline SA43400 & SA372-J & 736 & 861 \\
\hline G43300 & $\begin{array}{c}\text { DOT- } \\
\text { 3AAX }\end{array}$ & 641 & 786 \\
\hline
\end{tabular}

The 440A specimens were used in the undeformed state to measure the absorption of hydrogen by dislocations. Then, measurements of length change over time under ambient conditions could be isolated as being due to hydrogen absorption.

The 355 specimens were designed to measure the absorption of hydrogen by GNDs in addition to SSDs. They were subjected to a special bending procedure to create a volume of material whose dislocation density varied with an imposed strain from zero to 0.10 . This was done by bonding two specimens back-to-back and bending them $180^{\circ}$ on themselves around a $2 \mathrm{~mm}$ radius bar, then separating the samples by chilling them to make the bonding glue brittle enough to split them apart, thus permitting them to assume a stress-free position. Sandwiching the two strips permitted them to be separated at the neutral axis of bending at which no deformation occurs, producing two separate 
specimens, whose strain was an independent, controllable variable. Since the 355 specimens were only $250 \mu \mathrm{m}$ in thickness and the maximum strain created was 0.10 , the deformed portion of the specimen dimensionally approached that of an FPZ in martensitic steel. It was thought that the similarity of the deformed zone to that of a crack tip FPZ would provide valuable insights into the dynamic absorption of hydrogen in an FPZ. The plane strain in bending was similar to the stress state of a fracture toughness test to which the results from strip specimens would be applied.

Both the 355 and $440 \mathrm{~A}$ specimens were baked at $250{ }^{\circ} \mathrm{C}$ for four hours before any testing to remove most of the hydrogen. This temperature should remove deformationproduced vacancy clusters, while monovacancies should remain constant at a negligible level. The as-baked, low-hydrogen specimens were lightly sanded for several minutes with 600 grit material to remove the heat tint, whose thickness is less than one $\mu \mathrm{m}$, thus causing negligible surface cold work. During the repeated surface abrasion, they were then allowed to react with water vapor in ambient air at $25{ }^{\circ} \mathrm{C}$ and $60 \%$ relative humidity (1.0\% water vapor). Hydrogen absorption occurred during the abrasion and continued after abrasion until a full passive film formed. In the undeformed specimens, both 355 and $440 \mathrm{~A}$, hydrogen absorption is seen as a length change which continues until saturation. In deformed specimens, 355 only, deflections should be due to the variable absorption of hydrogen in the deformed section's dislocation gradient. This volume expansion was calculated from the deflection. High binding energy traps such as vacancies, grain boundaries, and incoherent precipitates should show little change in hydrogen content over this temperature range $\left(25-250{ }^{\circ} \mathrm{C}\right)$. Low binding energy traps such as coherent precipitates, though absent, should have negligible hydrogen content in this environment, so we can attribute all expansion to absorption of hydrogen by dislocations. However, we can measure the expansion effect of absorbed hydrogen on deformed material and impute a hydrogen concentration corresponding to an estimated dislocation density.

Measurements were performed with a Vinca DCLA 1205 contact digital micrometer with a resolution of $0.01 \mathrm{~mm}$. The standard deviation of all measurements, five per sample, was $\sim 0.025 \mathrm{~mm}$.

Both specimen types were measured for the effect of hydrogen expansion in the undeformed state, but to obtain the critical information of hydrogen absorption as a function of strain, a special procedure was conducted on the thinner 355 specimens. The bending procedure described earlier was designed to produce a deformation gradient through the thickness of the thin 355 specimens so the effect of deformation on hydrogen absorption could be quantified. The bending procedure resulted in a maximum 0.10 surface strain as calculated by the geometrically derived Eq. 3:

$$
\varepsilon=\frac{h}{r}
$$

where $\epsilon$ is the hoop strain due to the expansion from hydrogen absorption in the deformed zone, $h$ is the thickness, and $r$ is the radius of curvature. The strain in the width direction was zero except close to the edges, i.e., a plane strain condition existed across the broad face. The springback of the specimen bent in tension was approximately $25^{\circ}$. It is the deviation from the included angle of $25^{\circ}$, measured by specimen tip deflection, which allowed determination of the volume expansion strain due to the variation of hydrogen absorbed under various experimental conditions.

The specimens whose convex surfaces were deformed in tension would be expected to deflect to a more acute angle to the extent hydrogen uptake occurs. One portion of the bent strip was fixed and the other allowed to deflect freely. The deflection caused by hydrogen absorption could be measured and the deflection could be translated to a hoop strain which under plane strain would equal half the volume strain. It follows that the deflection can be geometrically related to the strain by Eq. 4:

$$
\varepsilon=\frac{h}{r} \times \frac{\Delta x}{L}
$$


where $\Delta x$ is the deflection and $L$ is the length of the strip from the deformed region $(10 \mathrm{~cm}$ in this case). For our specimens, a deflection of $1 \mathrm{~mm}$ equated to a linear strain of 0.1 , and the volume strain (change in volume divided by the initial volume) was twice the hoop strain since the specimens were geometrically constrained from contracting across the face.

In the first experiment, undeformed $440 \mathrm{~A}$ was de-hydrogenated by baking at $250{ }^{\circ} \mathrm{C}$ for four hours, quenched to room temperature, sanded to remove the heat-tint oxide, and allowed to equilibrate with ambient air as its change in length was monitored. The 440A specimens did not have sufficient surface chromium content to limit ambient corrosion, and red rust on the surface confirmed this.

The same procedures were used on the 355 specimens, but with the added variable of deformation as produced by the special bending described above. Also, the $355 \mathrm{did}$ have a passive film forming surface chromium level so repeated light abrasion was needed to produce saturating levels of hydrogen. The 355 specimens were also equilibrated at intermediate temperatures to observe the effect of lesser amounts of hydrogen removal based on the binding energy of dislocations. The expansion was compared to that which would be expected theoretically from reported experimental partial molar volumes, dislocation density, and trap filling percentage.

\section{Results}

Table 3 lists the experimental volume expansion and compares it to the theoretically expected volume expansion derived from the estimated dislocation density, temperature, and hydrogen fugacity. The expected change in volume due to hydrogen being absorbed by defects created by deformation is calculated by using Eq. 4 The agreement between expected expansion and measured expansion is reasonable and better in the 355 than in the $440 \mathrm{~A}$. Estimating dislocation density in $440 \mathrm{~A}$ is problematic due to the uncertainty of actual carbon in solution as-austenitized, which determines the concentration of carbon in the martensite and therefore dislocation density.

In addition to the dilation data, we were able to measure Deff, the effective diffusion rate, for 355 in the undeformed state by abrading just one side of a baked specimen. The time for hydrogen to reach the opposite surface $(t)$, which we approximated as the time to reach half maximum dilation, allowed $D_{\text {eff }}$ to be calculated by equation 5 :

$$
D_{\text {eff }}=\frac{x^{2}}{4 t}
$$

which for one sided diffusion with $\mathrm{x}=0.25 \mathrm{~mm}$ and $\mathrm{t}=100 \mathrm{~s}$ gave a Deff value of $6.25 \times 10^{-}$ ${ }^{10} \mathrm{~m}^{2} / \mathrm{s}$. This will be used later in the calculation of crack velocities.

Table 3. Change in volume due to hydrogen absorption.

\begin{tabular}{ccccccc}
\hline specimen & $\begin{array}{c}\Delta \mathrm{L} / \mathrm{L} \\
\mathbf{2 5}{ }^{\circ} \mathrm{C}\end{array}$ & $\begin{array}{c}\text { Expected } \\
\mathbf{\Delta L} / \mathbf{L}\end{array}$ & $\begin{array}{c}\Delta \mathrm{L} / \mathrm{L} \\
\mathbf{0}{ }^{\circ} \mathrm{C}\end{array}$ & $\begin{array}{c}\Delta \mathrm{L} / \mathrm{L} \\
\mathbf{5 0}{ }^{\circ} \mathrm{C}\end{array}$ & $\begin{array}{c}\Delta \mathrm{L} / \mathrm{L} \\
\mathbf{1 0 0}{ }^{\circ} \mathrm{C}\end{array}$ & $\begin{array}{c}\Delta \mathrm{L} / \mathrm{L} \\
\mathbf{1 5 0} \mathbf{~}^{\circ} \mathrm{C}\end{array}$ \\
\hline $\begin{array}{c}355 \\
\text { undeformed }\end{array}$ & 0.00030 & 0.00030 & - & - & - & - \\
\hline $\begin{array}{c}355 \\
0.01 \text { strain }\end{array}$ & 0.00260 & 0.00240 & 0.0036 & 0.0014 & 0.0010 & 0.0009 \\
\hline $\begin{array}{c}440 \mathrm{~A} \\
\text { undeformed }\end{array}$ & 0.00135 & 0.00090 & - & - & - & - \\
\hline $\begin{array}{c}440 \mathrm{~A} \\
\text { undeformed }\end{array}$ & 0.00135 & 0.00090 & - & - & - & - \\
\hline $\begin{array}{c}440 \mathrm{~A} \\
\text { undeformed }\end{array}$ & 0.00114 & 0.00090 & - & - & - & - \\
\hline
\end{tabular}




\section{Discussion}

\subsection{Experimental design}

While it would be ideal to conduct the experiments with the same materials for which both fracture toughness and hydrogen expansion data exist in the literature (especially 4340 and SA-372-L), such alloys were not available in sheet form for this study. Martensitic stainless steel alloys were available. This compromise was accepted because the chromium content which distinguished our specimens was not believed to have a material effect on any relevant mechanical property but would have a significant effect on hydrogen absorption if steps were not taken to avoid the hydrogen-generation diminishment caused by the stainless passive film curtailing surface reactions. The 355 alloy has a lower dislocation density than $440 \mathrm{~A}$ but has sufficient ductility in the asquenched state to be bent $180^{\circ}$. The $440 \mathrm{~A}$ alloy was too brittle to bend significantly but had the dislocation density and specimen size to maximize the expected growth in volume. The estimated dislocation density values were $5 \times 10^{11} / \mathrm{cm}^{2}$ for the 355 and $1.5 \times 10^{12} / \mathrm{cm}^{2}$ for the $440 \mathrm{~A}$ austenitized at a temperature to produce $0.40 \% \mathrm{C}$ in solution [13]. Using Hirth's saturation of five atoms of hydrogen per atomic plane for edge dislocations and one per atomic plane for screw dislocations [7], which we approximated as three per dislocation per atomic plane assuming equal edge and screw populations, we can calculate the saturation hydrogen level associated with these traps. Equation 2 provides the molar concentration of hydrogen for a given dislocation density. At room temperature, saturation of the traps equates to $\sim 15$ and $\sim 45$ wppm hydrogen for undeformed 355 and $440 \mathrm{~A}$, respectively. Hydrogen concentrations this high are not commonly reported for martensite. But lower levels are generally associated with either tempered or undeformed material and those not having the influence of hydrogen supplied by the passivation/oxidation reaction.

The more important distinction from the Hirth projections is that our experiments were conducted on unoxidized surfaces for which the hydrogen available for absorption comes from the oxidation/passivation reaction of the base alloy and water vapor. In alloy steels the hydrogen generation by atmospheric corrosion is sufficient to supply hydrogen to the underlying steel matrix in excess of the solubility as shown by the hydrogen permeation through steel membranes [17]. This bypasses Hirth's condition of pure hydrogen at varying pressures. Instead, the underlying reaction is the spontaneous formation of the mixed iron-chromium passive film from the reaction with water vapor which liberates atomic hydrogen on the alloy surface. To obtain a sufficiently non-passive film for the reaction with water vapor to proceed, the $440 \mathrm{~A}$ was left with a chromium depleted surface from the air austenitization so a passive film would not form, while the 355, which did not have a chromium depleted surface, was subject to repeated passive film removal sanding so that each successive passive film formation contributed hydrogen to the underlying base metal. It was observed that 10 to 20 sanding passes per side with 600 grit sanding block was required to provide sufficient hydrogen for maximum length change. This is consistent with the repeated generation of the approximately $6 \mathrm{~nm}$ passive film formed in similar stainless steels [18]. This regrettably circuitous procedure was necessitated by the lack of thin 4340 samples for which the atmospheric corrosion required to induce hydrogen saturation would have been simple.

Baking specimens to remove hydrogen trapped by dislocations would permit a hydrogen-free length to be measured and compared to the length after the specimens had equilibrated with hydrogen derived from the ambient atmosphere. The actual volume change derived from the length change would then permit us to assess changes in stress state which would occur in fracture process zones in fracture toughness tests, as we explain later.

Vacancies, to the extent they exist in the as-quenched state or have been created by deformation, will have been largely removed by the baking treatment, so we neglect them and attribute all expansion to absorption of hydrogen by all dislocation traps, cores, and strain fields. The binding energy of hydrogen to dislocations is complex, according to Hirth [7], and still not totally resolved, according to Gibala [19]. However, we can measure 
the expansion effect of absorbed hydrogen on deformed material and impute a hydrogen concentration corresponding to an estimated dislocation density even without knowing the subtleties of binding, since our treatment saturates all available traps. We can conclude from our experiments that hydrogen absorbed by defects does indeed cause significant lattice expansion and that it varies with level of deformation produced traps.

Having shown the existence of hydrogen-induced lattice expansion, we can proceed to the second part of the discussion, where we present the effect of this expansion on hydrogen embrittlement. We will present the model within the framework of the compact fracture toughness test and will change the alloy focus to 4340 for which much data is available, under the reasonable assumption that 4340 behaves similarly to its stainless martensitic counterparts from which we developed hydrogen expansion data.

\subsection{Effect of hydrogen-induced dilation}

The experimental results are hardly a discovery; and although they are not generally mentioned in discussion of hydrogen embrittlement, they are elementary. We can apply what was learned from simple expansion of sheet samples and sheet samples deformed in plane strain to plane strain fracture toughness specimens where expansion effects can be quantified. The implications of the dilation on crack mechanics are nearly as straightforward, as we will now discuss. In terms of classical mechanics, the size of the FPZ for a martensitic steel can be determined by Eq. 6, and can be represented schematically by Fig. 3A:

$$
\delta=\frac{K^{2}}{E \sigma_{y s}}
$$

where $\delta$ is the FPZ diameter, $K$ is the applied stress intensity, $E$ is the elastic modulus, and $\sigma_{y s}$ is yield strength.
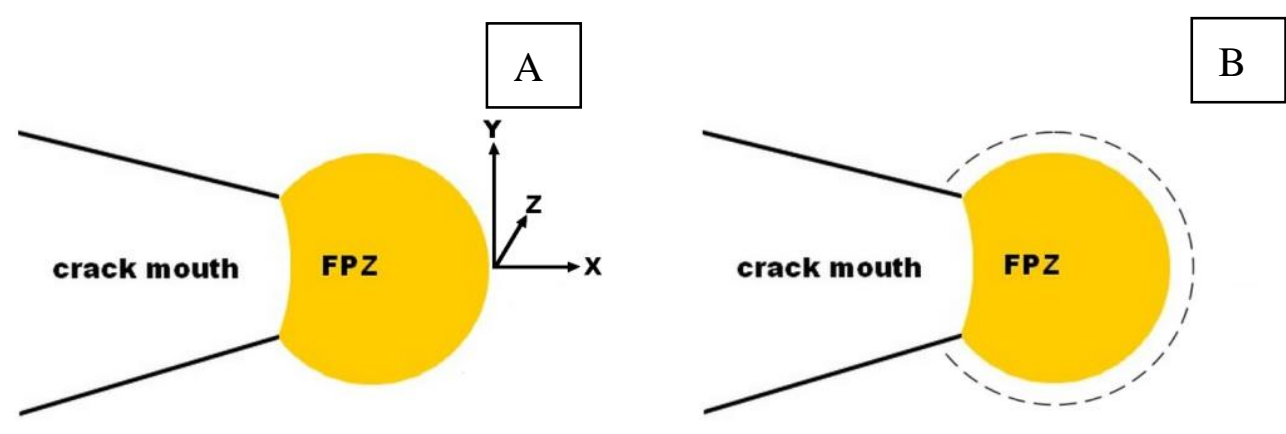

Fig. 3. A) Depictions of the fracture process zone (FPZ) region. All points in the FPZ except the free surface are in triaxial tension and the maximum is reached at the PMT where the coordinates are drawn. Deformation has ceased and stresses are in balance. B) Hydrogen has caused the FPZ to enlarge, as shown by a dashed line, thereby altering the stress state at the PMT.

The coordinates shown in Fig. 3A are located at the point of maximum triaxial stress (PMT) and serve as our reference system. As Eq. 5 indicates, increasing the crack mouth opening by increasing the load increases the size of the FPZ. However, the stress state at the PMT remains constant with $\left(\sigma_{y}-\sigma_{x}\right)$ slightly above the yield strength. The value of $\sigma_{y}$, the y-direction stress at the point of maximum triaxial stress, is commonly approximated as three times the yield strength in steels [20]. Except for the surface of the blunted crack opening, the entire FPZ is in triaxial tension after load application and arrest. The formation of a triaxial stress resisting crack opening is characteristic of elastic/plastic materials and is seen when the $\mathrm{z}$ dimension is adequate to provide plane strain. The level of deformation is approximated to vary linearly in the $\mathrm{x}$ direction, so it is close to the base level of the surrounding matrix at the PMT and increases linearly from the PMT to the crack opening. 
In an actual rising-stress fracture toughness test in the absence of hydrogen, a rising stress intensity increases the FPZ size until fracture begins at the crack tip by the same failure mode seen under plane strain conditions. The FPZ triaxial zone itself is a fundamental characteristic of elastic/plastic behavior. In thick specimen plane strain, resistance of the FPZ to volume expansion confines the deformation to a small zone, in contrast to a thin sheet where there is no constraint to lateral contraction and the deformation zone is much larger in extent for a given load per unit width. In the absence of hydrogen, in either the thin or thick specimen, failure occurs at the crack tip opening when the elongation limit of the material is reached at the crack tip surface. In a thick specimen the triaxial stress state in the FPZ is mechanically precarious. It cannot tolerate a volume change in the FPZ without crack destabilization. The three orthogonal stresses are above the yield strength but are balanced so that plastic flow has stopped at crack arrest, leaving $\left(\sigma_{y}-\sigma_{x}\right)$ everywhere in the FPZ above the yield strength. But by the Tresca flow criterion we know that if the algebraic sum of the maximum and minimum principal stresses $\left(\sigma_{y}-\sigma_{x}\right)$ increases, plastic flow will resume. Now, it becomes obvious why we concern ourselves with hydrogen-induced expansion. The balance of stresses is established instantly with deformation, but after deformation hydrogen enters the FPZ to bind with the defects which the deformation has created. Expansion occurs, and the stress balance is lost. Our model characterizes how the crack responds to this destabilization.

Our focus is on the FPZ. If it expands relative to the volume around it, as depicted in Fig. 3B, stress states are altered. In a crack under stress any increase in the difference between $\sigma_{\mathrm{y}}$ and either $\sigma_{\mathrm{x}}$ or $\sigma_{\mathrm{z}}$ will cause further deformation. Hydrogen expansion would provide that change in stress because the expansion occurs almost entirely between the crack tip surface and the PMT. This asymmetry of the hydrogen lattice expansion is key. How can we quantify the destabilization which would occur when the stress balance is altered? The FPZ itself can be considered an Eshelby zone [21]: a deformed zone nearly surrounded by a less-deformed matrix. This assumption simply allows us to approximate the interfacial stress component acting normal to the PMT in the $x$ direction $(\sigma \mathrm{H})$, caused by the expansion of the FPZ lattice to be one half of the expansion volume strain times the bulk modulus, $160 \mathrm{GPa}$, according to [20]. The FPZ "pressurizes" with absorbed hydrogen and expands. The surrounding matrix recoils until the stress at the interface between the two is equal. So, at the PMT, which borders the expanding FPZ Eshelby zone, the crack opening stress $\left(\sigma_{\mathrm{y}}\right)$ increases marginally because of the expansion of the FPZ, and some crack opening load is shifted beyond the PMT. The effect on $\sigma_{z}$. at the PMT is also negligible. The critical effect is on $\sigma_{x}$ because the addition of the compressive hydrogen expansion stress to the existing tensile $\sigma_{x}$ results in a net decrease in $\sigma_{x}$, increasing the difference between $\sigma_{y}$ and $\sigma_{x}$. Eq. 7 below expresses the relationship between hydrogen expansion stress and $\sigma_{x}$ :

$$
\sigma_{x}=\sigma_{x i}+\sigma_{H}
$$

where $\sigma_{x}$ is the post-expansion stress acting on the PMT, $\sigma_{x i}$ is the pre-expansion stress, and $\sigma_{H}$ is the stress induced by the hydrogen expansion after the Eshelby factor has been considered. $\sigma_{x i}$ is tensile and approximately twice the yield strength while $\sigma_{H}$ is compressive and therefore causes a decrease in $\sigma_{x}$.

A small change in $\sigma_{x}$ means additional plastic flow and incremental crack mouth opening. A sufficiently large change will cause the difference between $\sigma_{y}$ and $\sigma_{x}$ to exceed the material's tensile strength. In this way it is possible for hydrogen to cause "embrittlement" without altering the inherent mechanical properties of the material. The recognition of the importance of the radial (x direction) component of the expansion stress is the main factor of the hydrogen lattice dilation model, HLD.

Hydrogen does not need to cause huge stresses to cause plastic flow or even decohesion; it simply must marginally lower $\sigma_{x}$. The stress decrement required to cause decohesion is the difference between the yield strength and the tensile strength, since the 
PMT and the rest of the FPZ is on the verge of further yielding. Lesser reductions in $\sigma_{x}$ will not cause brittle failure but can cause enhanced plastic flow sufficient for failure in a different way, explained later.

The effects of hydrogen on the stress state define the proposed model, which we call the hydrogen lattice dilation model (HLD). It is nothing more than the dilation of the lattice due to hydrogen being absorbed after deformation by the defects formed during deformation. The stresses created by the hydrogen absorption can thus be superimposed on those of classical stress analysis without hydrogen present.

The magnitude of the HLD stresses can be estimated from both theory and our experimental results. In the 355 alloy, as shown in Table 2, a 0.1 (10\% hoop strain) deformation would result in an estimated increase to $5 \times 10^{12} / \mathrm{cm}^{2}$ in the dislocation density, which if saturated with hydrogen would be the obvious cause of the 0.0045 volume increase. This in turn results in a net change in $\sigma_{x}$ of $360 \mathrm{MPa}(0.0045 \times 160 \mathrm{GPa}$ bulk modulus times the one half Eshelby factor). Since this $360 \mathrm{MPa}$ is typical of the difference between the yield and tensile strengths of martensitic steels, it is logical that hydrogen-induced expansion would have the capacity to cause failure since it can cause $\left(\sigma_{\mathrm{y}}-\sigma_{\mathrm{x}}\right)$, to reach that critical level for failure.

\subsection{The hydrogen lattice dilation model}

If we apply the mechanics of the lattice expansion, as driven through hydrogen absorption by dislocations to a series of fracture toughness tests at room temperature (under consistent levels of hydrogen activity and constant load), it is possible predict certain events and compare them to literature values. We note that the absorption of hydrogen requires that atomic hydrogen diffuse from the surface to the newly created traps. There is also an elastic strain gradient which would increase the lattice solubility of hydrogen in the FPZ, but even with this strain the solubility in normal lattice interstitial sites is negligible. The hydrostatic strain enhances the binding energy of hydrogen to defect traps as well, providing an additional binding energy $\sigma_{h} V_{H}$, where $\sigma_{h}$ is the hydrostatic stress and $V_{\mathrm{H}}$ is the partial molar volume of hydrogen, taken as $2.0 \mathrm{~cm}^{3} / \mathrm{mol}$ [10]. Whether this enhanced binding energy is significant depends on the marginality of trap saturation for a given set of conditions.

In the broadest sense the behavior we wish to understand is described by Fig. 4 [8], where $\mathrm{K}_{\text {тна }}$ is the threshold stress intensity determined by fixed crack mouth opening displacement (CMOD), and $\mathrm{K}_{\mathrm{JH}}$ is that determined by slow rising CMOD in the terminology of reference [8]. In our terminology $\mathrm{K}_{\text {THa }}$ is $\mathrm{K}_{\text {th2, while }} \mathrm{K}_{\mathrm{JH}}$ is what we call $\mathrm{K}_{\text {th1 }}$. This dual behavior, different $K_{\text {th }}$ values depending on test method, has been treated as paradoxical. We will show that is a natural consequence of HLD and a demonstration of our contention that two modes of hydrogen embrittlement arise from a single mechanism. Fig. 4 comes from a comprehensive study [8] that maps hydrogen embrittlement in unprecedented detail, which we will repeatedly refer to as a test of our model.

The yield stress at the intersection of the two curves in Fig. 4 is important in that it should be constant regardless of hydrogen fugacity and temperature for a given alloy system because both sides of the threshold defining equation are driven by the same hydrogen traps. We have added what HLD predicts for Kth1 and $K_{\text {th2 }}$, to Figure 4 and we will later show how these were calculated. 


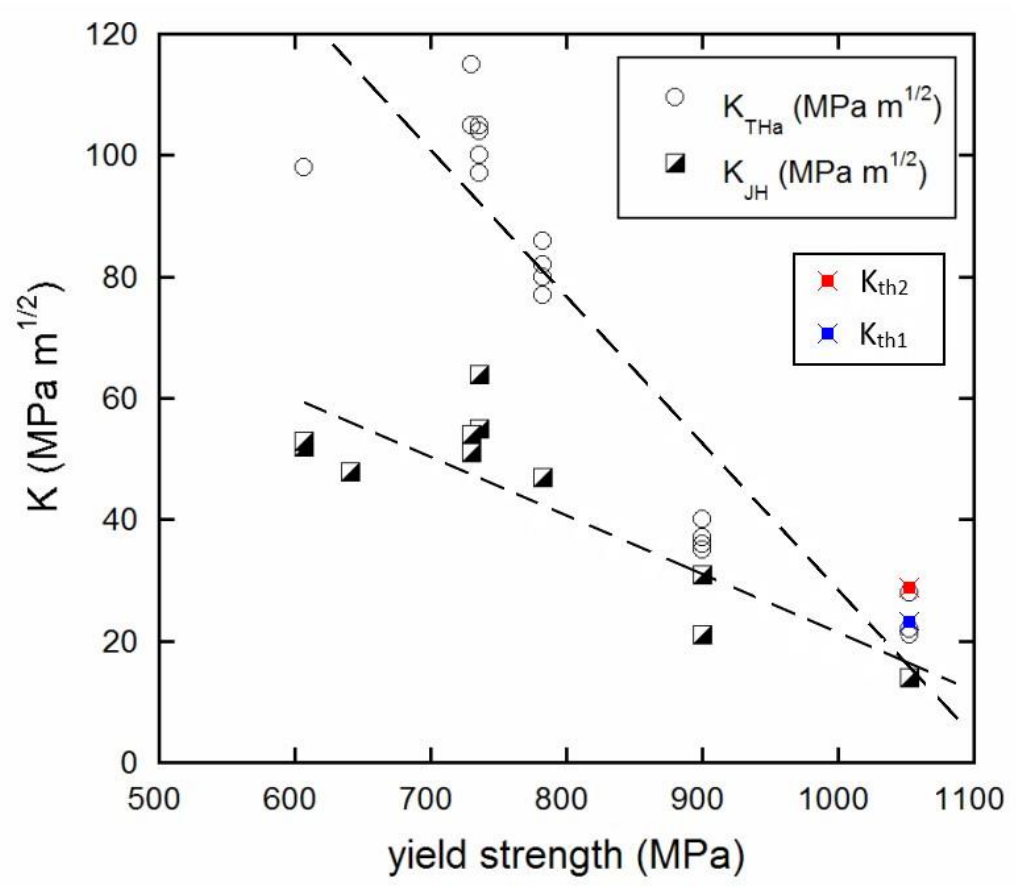

Fig. 4. The variation of the differing $K_{\text {th }}$ levels as measured by two testing methods, from [8]. HLD predictions of both $K_{\text {th } 1}$ and $K_{\text {th2 }}$ were added to the original graphic. Dashed lines are least-squares fits and are also. The alloys from this study are DOT-3T, DOT3A, SA372-J, and SA372-L, whose full compositions and mechanical properties provided elsewhere [8]. Tested in $100 \mathrm{MPa}$ hydrogen. HLD predicted values for 1050 MPa yield strength are shown.

\subsubsection{Rising CMOD tests and HE1 mode}

Here we describe the logic behind the HLD model. In a rising CMOD test, the compliance curve run without hydrogen is linear from zero until the beginning of crack advance. The HLD model predicts that the FPZ size due to hydrogen absorption would be enlarged at the same K giving a greater CMOD. We posit that the FPZ diameter in the presence of hydrogen is given by:

$$
\delta=\frac{K^{2}}{E\left(\sigma_{y s}-\sigma_{H}\right)}
$$

where $\sigma_{H}$ is the $x$ direction stress at the PMT cause by the hydrogen lattice expansion. This difference in FPZ size due to hydrogen would be observable by unloading a specimen just before crack advance, where it would be seen as an excess residual crack tip opening, which has been reported, but attributed to flow outside the crack area [8]. The objective of the rising CMOD test is to identify the load at which crack advance begins. The critical load by our model is that at which rising $\mathrm{K}$ has created sufficient deformation for the hydrogen-induced change in $\sigma_{x}$ to reach $\gamma \sigma_{y s}$ (where $\gamma$ is Poisson's ratio). When this occurs, plane strain no longer exists at the PMT. At this point the stress in the y direction $\left(\sigma_{y}\right)$ at the PMT goes to simply the uniaxial flow stress. Triaxial stress no longer exists to prevent crack opening. If a triaxial stress cannot be maintained, the FPZ elongates into a plastic zone where strain varies from a maximum at the crack mouth to the far field value at a large distance from the crack mouth. The absence of a constraint to plastic flow, which the triaxial zone had provided, means the crack would now behave as a crack in a thin sheet, where failure occurs by stable tearing at the crack apex. Thus, the criterion in the HLD model for what occurs at $K_{\text {th }}$ in a rising CMOD test is simply Eq. 9:

$$
\sigma_{\mathrm{H}}=\gamma \sigma_{\mathrm{ys}}
$$


When the rising stress intensity reaches $K_{t h 1}$, the collapse of the triaxial stress will cause an increase in the crack tip opening without, at first, a true change in crack length, an extreme version of crack blunting [22]. This is because the collapse of the triaxial zone causes a major redistribution of the $\sigma_{\mathrm{y}}$ distribution and possibly an abrupt change in crack tip opening as the constraint of the triaxial stress state is lost. True crack advance occurs only after the collapse and the absorption of hydrogen by the new, much deeper plastic zone, which lacks a plastic flow-stopping triaxial stress state. When the plastic zone has grown, post-collapse, to a sufficient level, the crack mouth opening reaches its inherent limit and fails by tearing. That limit will either be the crack mouth opening seen in the absence of hydrogen at the plane-strain fracture toughness $\left(\mathrm{K}_{1 \mathrm{c}}\right)$ or possibly some lesser amount of elongation. The degree to which elongation of the crack tip opening is diminished, versus that seen in a test run without available hydrogen, is the amount which must be provided by a true embrittlement or property-degrading mechanism. If it exists, and this has not been shown, it would be the elusive influence of enhanced plasticity hydrogen embrittlement models.

After $K_{\text {th1 }}$ is reached the information from a rising CMOD test is of less value than that of the constant load test, in terms of analysis of crack velocity. At sufficiently slow CMOD increase rates, crack velocity may be simply determined by crack mouth opening rate. In fact, if the imposed crack opening were stopped, then crack growth must also stop when equilibrium hydrogen content is reached; this behavior is the definition of stable cracking and an implied condition of the HLD model.

The cracking seen under the above slow rising CMOD test conditions is in the tearing mode, which we call HE1. It predicts that what we will now call Kth1 decreases with increasing yield strength, as seen in a study of 4340 type steels tempered to various strength levels (Fig. 4) [8]. At a given $\mathrm{K}, \sigma_{H}$ in these alloys increases with yield strength, while yield strength increases with the square root of the dislocation density, as in the Bailey-Hirsch relationship. Therefore, as the quantity $\gamma \sigma_{y s}$ increases, a lower applied $\mathrm{K}$ is required to reach the dislocation density to make $\sigma_{\mathrm{H}}=\gamma \sigma_{\mathrm{ys}}$ (Eq. 8). The tearing mode is associated with plane stress. Again, cracks propagated in the tearing mode are stable, i.e., increments of crack mouth opening are required to cause an increase in crack length. This has been observed to be a characteristic of rising CMOD tests in hydrogen [6]. Incidentally, the crack front should be straight across the specimen as the stress state is the same across the crack front.

The cracking mode which we call HE1 is very interesting insofar as it can be viewed as just the opposite of embrittlement. It is manifest as an increase in apparent ductility by the increased crack zone plastic flow. The crack tip and mouth do open more at a given load because of the change in stress state caused by the hydrogen-induced expansion. However, there is no evidence that the strain at the crack tip at fracture changes from that which would occur without hydrogen. If it did, it would validate HELP-type models, and more sophisticated experiments are needed to determine this. But what is clear is that the load bearing ability of the material is reduced by this form of hydrogen embrittlement. At the same time there is a benefit in that the material accommodates imposed deformation without rapid crack advance. So, HE1 is a benign form of hydrogen embrittlement, which could be considered as acceptable for design purposes if its low crack advance rates did not compromise its intended use, as the more rapid HE2, which we discuss next, certainly can.

\subsubsection{Fixed CMOD tests and HE2 mode}

While much research is focused on the HE1 mode of hydrogen embrittlement as seen in rising CMOD tests, the fixed CMOD test paints a much different picture, though one which is interpreted equally well by HLD. In a fixed-opening CMOD test in which hydrogen is supplied by the environment, the triaxial stress zone is intact before hydrogen entry. The triaxial zone has not collapsed as in a rising CMOD test. It is a defining characteristic of HE2 that above a certain stress intensity, HE2 cracking proceeds at a constant velocity regardless of stress intensity. If we examine this lowest stress intensity 
at which the constant velocity occurs, it is at a different $\mathrm{K}_{\text {th }}$ than found in rising CMOD as seen in Fig. 4. At this smallest $\mathrm{K}$ at which $\mathrm{K}$-independent crack advance occurs, the HLD model holds that the same lattice expansion mechanism is at work but in a different mode. We will designate the $K_{\text {th }}$ seen in rising stress intensity tests as $K_{\text {th1, }}$ while we will call the one seen at fixed CMOD intensity $K_{\text {th2. }}$

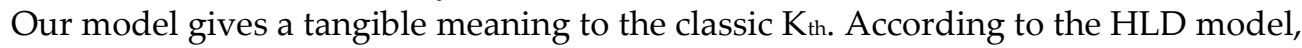
$K_{\text {th2 }}$ occurs when deformation in the FPZ produces a hydrogen trap density for which the equilibrium $\sigma_{H}$ has just the critical value to cause the principal stress $\left(\sigma_{\mathrm{y}}-\sigma_{\mathrm{x}}\right)$ at the PMT to equal the fracture stress in uniaxial tension. The critical value of $\sigma_{\mathrm{H}}$ is simply $\left(\sigma_{\mathrm{tts}}-\sigma_{\mathrm{ys}}\right)$, where $\sigma_{\mathrm{tts}}$ is the true tensile strength. This can only occur when the triaxial stress configuration is stable, which is why it typically does not occur under rising CMOD. In which the triaxial stress zone has collapsed. The failure is brittle at initiation since the failure is caused by immediate overload in triaxial tension. It should show essentially no plastic flow except that which had occurred during the deformation which preceded hydrogen entry. It will first meet the failure criterion, Tresca or Von Mises, at the PMT after which it will propagate to the crack mouth. The zone beyond the PMT will then form a new FPZ with a new triaxial stress zone. The equation for the failure criterion for HE2 is thus:

$$
\sigma_{H}=\sigma_{t t s}-\sigma_{y s}
$$

If decohesion were to play a role in the fracture, it would be seen as fracture occurring at a level of $\sigma_{\mathrm{H}}$ less than $\left(\sigma_{\mathrm{tts}}-\sigma_{\mathrm{ys}}\right)$. The loss in tensile strength of the material would be due to hydrogen-induced decohesion. However, we assume that the hydrogen lattice expansion alone may be sufficient to cause failure without any effect of lessening cohesive strength. At a greater stress intensity than $K_{\text {th2 }}$, the critical $\sigma_{H}$ would be generated closer to the mouth than the PMT due to the increase in dislocation density, as illustrated in Fig. 5:

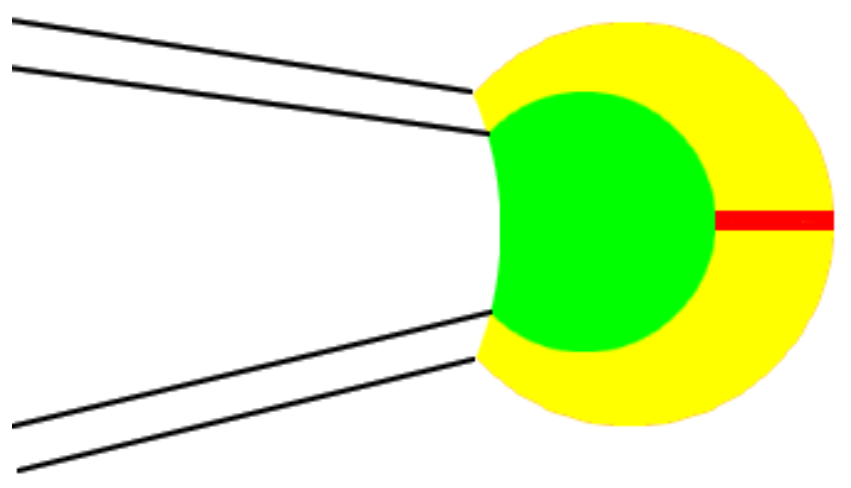

Fig. 5. The FPZ at $\mathrm{K}_{\text {th2 }}$ in green and at a greater stress intensity in yellow. The red line is the locus of all points which satisfy Eqs. 10 and 11.

The excess hydrogen in the FPZ over that in the matrix, $\Delta[\mathrm{H}]$, is that bound to newlycreated defects, assumed to be principally dislocations, whose saturation level is determined by hydrostatic stress, hydrogen fugacity, and temperature. The equation describing the fracture criterion is: 


$$
\sigma_{\mathrm{H}}=\beta \Delta[\mathrm{H}][0.28] \mathrm{B}=\sigma_{\mathrm{tts}}-\sigma_{\mathrm{ys}}
$$

where $\beta$ is an Eshelby shape factor roughly equal to 0.5 , $\mathrm{B}$ is the bulk modulus, and 0.28 is the ratio of the partial molar volumes of [hydrogen in steel]to[steel]. $[\mathrm{H}]$ is the excess hydrogen in the FPZ over that in the undeformed matrix. We note that in other alloy systems, such as alloys with the addition of precipitation hardening, Eq. 11 would still apply but $[\mathrm{H}]$ would be a more complex function of dislocation density, vacancy population, and density and strength of traps at coherent interfaces.

The locus of failure is shown in Fig. 5 as a red line. All points along this line satisfy

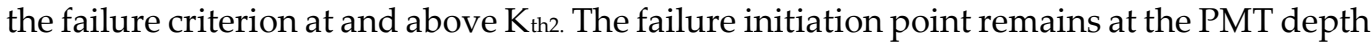

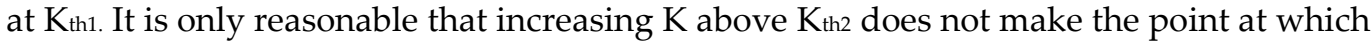
fracture occurs at $K_{\text {th2 }}$ less vulnerable, so the initiation point does not remain at the PMT at a stress intensity above $K_{\text {th2. }}$ In fact, if the defect gradient is linear, the fracture initiation will occur at the same critical distance from the crack tip surface, $\lambda$, as it does at $K_{\text {th2 }}$. Hydrogen does not need to diffuse all the way to the deeper PMT of the higher K. (Internal hydrogen rather than external hydrogen will change this.) This constant depth of crack initiation will, of course, dictate that the crack velocity stays constant with increasing stress intensity. The HE2 mode differs from HE1 in that it is discontinuous and is accompanied by minimal plastic flow because it occurs under triaxial stress, while HE1 occurs under plane stress tearing. In HE2 the crack initiates at some point on the red line and progresses to the crack mouth. Along its path it passes through highly deformed material. Thus, fractographic studies will show a brittle initiation and many signs of deformation along the fracture path and adjacent to the path due to the plastic strain history of the material the crack is penetrating.

Because the dislocation density coincides with and causes an increase in strength, $\mathrm{OH}$ increases with increasing yield strength, requiring less deformation to reach a critical $\sigma_{\mathrm{H}}$ for HE2 with an additional variation due to $\left(\sigma_{\mathrm{tts}}-\sigma_{\mathrm{ys}}\right)$ typically decreasing with yield strength, providing the upper curve in Fig. 4 with the lower curve governed by Eq. 4 for HE1 behavior. The intersection of the two curves will then occur according to HLD at the strength at which $\gamma \sigma_{y s}=\left(\sigma_{t t s}-\sigma_{y s}\right)$. To the extent these terms equal $\sigma_{H}$, the HLD model is expressed and validated. No model could be found in the literature which has successfully addressed the root cause of the two curves in Fig. 4 or the cause of their intersection. We will later discuss how this most characteristic indicator of an alloy system's hydrogen embrittlement behavior can be a predictor of hydrogen embrittlement-resistant alloy development, which may provide the most tangible benefits of this study.

\subsection{Application of the model to macroscopic behavior}

The expected value of a model is its agreement with observed behavior and its ability to predict behavior where observations do not exist. We will now attempt to verify both. Our concerns center primarily on threshold stress, crack velocity and fracture surface. A good way to test the model's predictions is to compare its predictions to the actual data [8]. Fig. 6 shows some of that study's data, which in this case were derived from fixed CMOD tests in high pressure hydrogen. Some of their results show many of the characteristic events that our model is uniquely able to rationalize. An analysis of these results follows.

Fig. 6 shows the fixed CMOD test results in 100 MPa pressure hydrogen with $K_{\text {thi }}$ values noted by arrows. The study [8] found that there is a stress intensity, $K_{\text {thi, }}$ above which cracking is independent of K. Cracking initiated at higher K could, however, continue at a stress intensity below $\mathrm{K}_{\text {thi. }}$. The study showed that the $\mathrm{K}$ at crack arrest, i.e., the traditional $\mathrm{K}_{\mathrm{th}}$, is not the same as $\mathrm{K}_{\text {thi. }}$. The HLD model concurs with that and predicts that crack arrest marks the beginning of HE1 and the end of HE2. The FPZ collapse occurs at crack arrest and initially causes crack mouth opening without crack advance as the triaxial stress state collapses, but which can be seen experimentally as crack growth, possibly temporarily increased, before true crack growth by HE1 commences with 
hydrogen diffusion to a greatly expanded plastic zone. This causes the crack tip to continuously open, eventually reaching failure.

In Fig. 6 we calculate and indicate with added red lines the expected crack velocity ( $V$ is the same as da/dt) for HE2-based crack advance based on the time for hydrogen to fill the FPZ, which we express as:

$$
V=\frac{4 D_{e f f} \sigma_{y s} E}{K_{t h 2}^{2}}
$$

For the top red line (alloy SA372-L with $1051 \mathrm{MPa}$ yield strength) we calculated V from Eq. 12 as $0.0006 \mathrm{~m} / \mathrm{s}$ for $D_{\text {eff }}=6.25 \times 10^{-10} \mathrm{~m}^{2} / \mathrm{sec}$, our experimentally determined effective diffusivity for this dislocation level, which is shown on Fig. 6. This is consistent with our model's constant depth of crack initiation. The level of Kth2 itself varies with defect, primarily dislocation, density, for which we have no data from [8], and therefore also varies with yield strength. For our purposes knowing the distance to the PMT as a function of yield strength was sufficient to calculate crack velocity. Since we do not have data on dislocation densities as a function of $K$ or even of strain for these alloys, nor $\left(\sigma_{t t s}-\sigma_{y s}\right)$, we cannot predict $K_{\text {th2 }}$ itself, with the exception of the strength level of our experiments, 1050 MPa yield strength so the crack velocities (shown by the red lines) are set at $K_{\text {thi, }}$ which we consider $K_{\text {th2. }}$. We can however predict $\mathrm{V}$ knowing $\mathrm{K}_{\text {th2 }}$ or we can predict $\mathrm{K}_{\text {th2 }}$ knowing V. Knowing dislocation density would permit either to be calculated, as we have shown at the $1050 \mathrm{MPa}$ yield strength level.

The red lines indicate $\mathrm{V}$ as predicted by experimental Kthi. Fig. 6 shows the transition from HE2 to HE1, where the lines change from red to blue. The blue lines were calculated in this study and added to the original figure from [8]. The blue dashed lines are calculated from Eq. 13 and begin at $K_{\text {thi. }}$ Moving toward lower values of $K$, these calculated dashed lines become dotted lines after the experimental data from [8] depart from the curve and drop toward zero V. They then continue to the actual experimental values of Kth1, which are indicated by blue circles (which have also been added to the figure from [8]). This transition from HE2 to HE1 occurs when the applied $\mathrm{K}$ is too low to create a defect density sufficient to generate a $\sigma \mathrm{H}$ which can initiate fracture at the PMT yet is well above $\gamma_{\text {ys. }}$. When this occurs, the triaxial stress collapses as it does in the HE1 mode seen in rising CMOD tests. Without a PMT the FPZ transforms into the elongated plastic zone extending characteristic of plane stress. If a specimen is loaded to a level between $K_{\text {th1 }}$ and $K_{\text {th2 }}$, there will be a time, possibly quite extended, after this collapse during which there is crack tip opening without true crack growth. This is the observed incubation time, which is characteristic of HE1 behavior [8]. In HE2 the time delay is much smaller, as little as a fraction of a second, while hydrogen diffuses a distance less than or equal to the FPZ depth. In HE1 the collapsed plastic zone is much deeper, and hydrogen diffuses into this zone causing the blunted crack tip to grow until it reaches the limiting failure opening, which as stated before will be the opening at K1C, or less, if hydrogen does indeed reduce ductility. The HLD model leaves room for hydrogen enhanced local plasticity to contribute to HE1, but in martensite its contribution seems minimal as the hydrogen stress appears sufficient to cause failure.

The declining experimental crack velocity from [8] in Figure 6 drops toward zero before reaching $K_{\text {th1 }}$. In [8] this is attributed to specimen plastic deformation away from the crack. We alternatively speculate that it is from the enlarged plastic zone encroaching on the extreme end of the specimen. This would account for the mismatch between values of $K_{\text {th } 1}$ as measured by rising and fixed CMOD tests. We posit that it is the lack of specimen length in the fixed CMOD tests causes a premature velocity fall to zero before $\mathrm{K}_{\text {thi }}$ is reached. 
The additional blue dashed lines indicate the crack velocity theoretically varying with $\mathrm{K}^{6}$. The lines are drawn to initiate at the experimental value of $\mathrm{K}_{\text {th1 }}$ and connect to $K_{\text {th2 }}$ indicated by equation 13. HLD would predict that the crack velocity would be determined by the time it would take hydrogen to fill a plastic zone in which triaxial stress has collapsed to the point at which crack tip opening reaches the fracture strain, which it does just below $\mathrm{K}_{\text {th2. }}$. We propose that the same plastic zone dimensions must be achieved at lower $\mathrm{K}$ levels to cause further crack advance, i.e., the crack tip opening remains constant at failure at all $\mathrm{K}$ levels during HE1. We further propose that the crack tip opening at a given $\mathrm{K}$ between $\mathrm{K}_{\text {th2 }}$ and $\mathrm{K}_{\mathrm{th}}$, which is initially that by given by equation 7 , must grow to the maximum $\delta$ characteristic of failure without hydrogen for crack advance to occur. We can express this as:

$$
V=V_{k t h 2} \frac{K^{6}}{K_{t h 2}^{6}}
$$

This proportionality of $\mathrm{K}^{6}$ derives from equation (14) in which the time to fill a plastic zone, $t$, by diffusion is determined by the dislocation density times the plastic zone volume, Volp, and the square of the plastic zone depth divided by the hydrogen flux which is controlled by $\mathrm{D}$, the diffusion rate and the crack tip opening, $\delta$, and a geometric factor, $\mathrm{C}_{0}$, as a constant,

$$
t=\frac{\rho V o l_{p} \delta^{2}}{\delta D_{e f f}} C_{0}
$$

Because $\mathrm{Vol}_{\mathrm{p}}$ is proportional to $\delta^{2}$ and $\delta$ is proportional to $\mathrm{K}^{2}$ equation 14 can be simplified with a different constant, $\mathrm{C}_{1}$ to:

$$
t=\frac{\rho K^{6}}{D_{\text {eff }}} C_{1}
$$

We do not show a red line for the lowest curve, that of the $641 \mathrm{MPa}$ yield strength alloy. In our opinion its failure mode is fully HE1. 


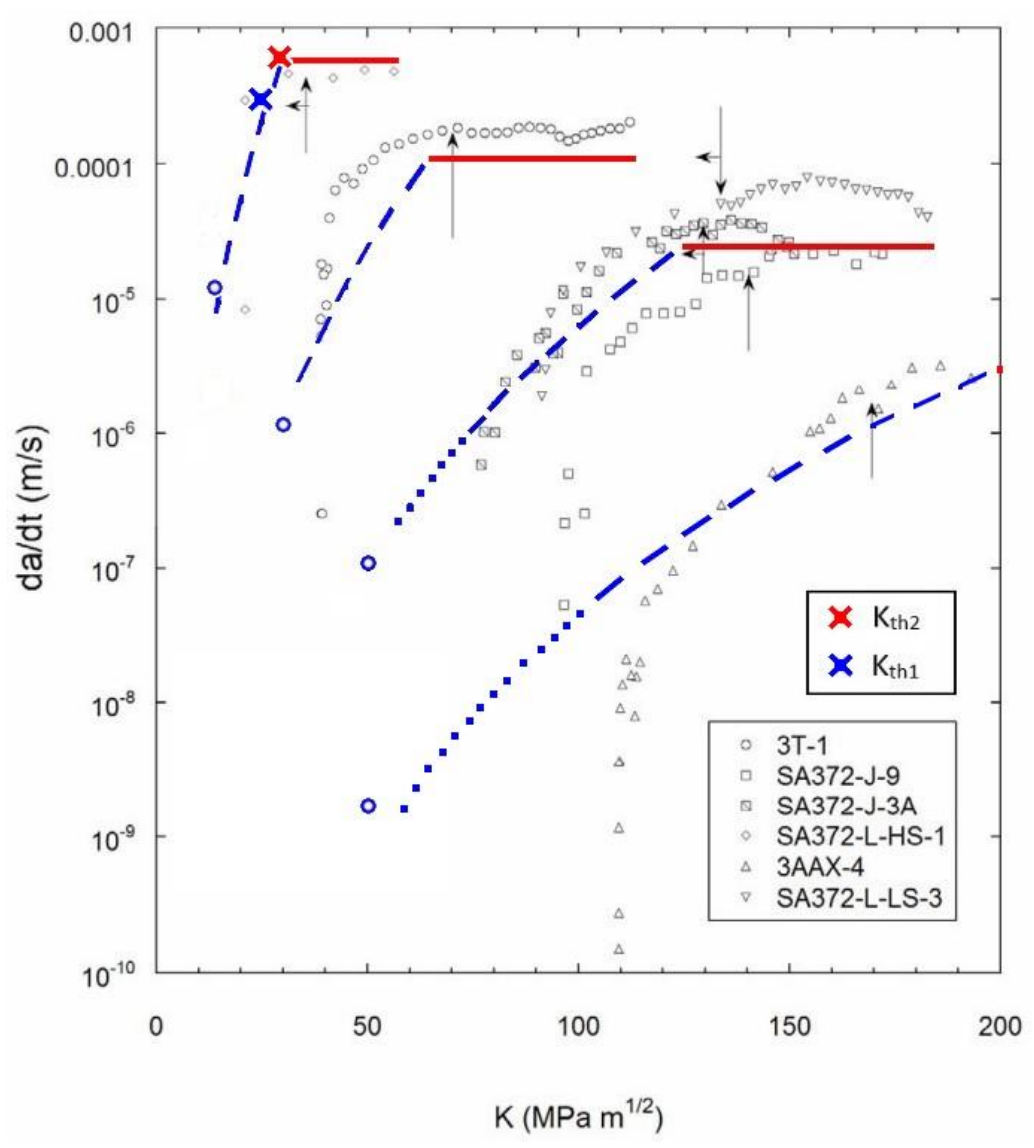

Fig. 6. Crack growth rate plots taken from [8] The red and blue colored lines, the points and legend for $K_{\text {th1 }}$ and $K_{\text {th2 }}$, and the blue circles have been added to the figure, as explained in the text.

The validity of the HLD model is supported by the fit between the shape of the four curves in Fig. 6. The connection of the calculated blue lines from experimental $K_{\text {th } 1}$ and $\mathrm{K}_{\text {th2 }}$ is strong support. The intersection of the blue and red line at the calculated HE2 velocities is further strong support. However, the strongest support comes from using the experimental data from alloy 355 to predict the fracture toughness behavior of SA372-LHS-1 in high pressure hydrogen. The mechanical properties of SA372-L-HS-1 parallel those of 355, both having approximately $1050 \mathrm{MPa}$ yield strength and $1300 \mathrm{MPa}$ true tensile strength. This alloy has a typical $\mathrm{K}_{1 \mathrm{c}}$ of $125 \mathrm{MPa}^{1 / 2}$ [23], giving it a crack tip opening by Eq. 3 of $~ 0.020 \mathrm{~mm}$ at failure which in turn corresponds to a typical true strain at fracture of 0.5 for this alloy, i.e., $50 \%$ RA in tension. Our similar 355 required a strain of 0.10 to generate an expansion stress of $360 \mathrm{MPa}$ which would occur at $\mathrm{K}=41 \mathrm{MPa} \mathrm{m}^{1 / 2}$. HLD requires a $250 \mathrm{MPa}$ hydrogen expansion stress for failure (1300 MPa minus 1050 $\mathrm{MPa}$, equation 6). To find the $\mathrm{K}$ level at which this occurs we need to find the strain which produces this hydrogen stress and convert that to a $\delta$. Equation 16 states that, based on the fact that both strain and dislocation density are related to stress, they are related to each other. Stress is related to strain by the work hardening exponent, here assumed as 0.25 while stress is proportional to the square root of dislocation density. This permits us to write equation 16 below

$$
\frac{\varepsilon_{1}^{0.25}}{\varepsilon_{2}^{0.25}}=\frac{\rho_{1}^{0.5}}{\rho_{2}^{0.5}}
$$

The hydrogen expansion stress ratio we are concerned with is $250 \mathrm{MPa} / 360 \mathrm{MPa}$ making the right-hand side of the equation equal 0.70 , from which we can calculate occurs at $\mathrm{K}=$ 
$29 \mathrm{MPa} \mathrm{m}^{1 / 2}$. by solving for strain and converting that to a $\delta, \sim 0.004 \mathrm{~mm}$ from which we use equation 6 to determine $\mathrm{K}$.

We have just calculated that at a yield strength of $1050 \mathrm{MPa}$, the predicted value for $\mathrm{K}_{\text {th } 1}$ is $29 \mathrm{MPa} \mathrm{m}^{1 / 2}$ versus the experimentally observed approximately $28 \mathrm{MPa} \mathrm{m}^{1 / 2}$. The predicted value for $\mathrm{K}_{\text {th2 }}$ must be calculated differently, since the same applied $\mathrm{K}$ as determined by having the same CMOD produces a larger crack tip opening, $0.005 \mathrm{~mm}$ versus $0.004 \mathrm{~mm}$ per Eq. 4 , and therefore a different required crack tip strain, 0.084 versus 0.067 . So, the dislocation density and, therefore, the hydrogen stress are estimated to be $25 \%$ more than $250 \mathrm{MPa}$ needed for $\mathrm{K}_{\mathrm{th}} 1$, or $315 \mathrm{MPa}$. So, under atmospheric pressure the expected $\mathrm{K}_{\text {th } 1}$ would be $28 \mathrm{MPa}^{1 / 2}$. However, the rising CMOD test was performed in a $100 \mathrm{MPa}$ atmosphere, independent of the imposed K. Therefore, our target hydrogen stress under these circumstances must be reduced by $100 \mathrm{MPa}$ to a hydrogen stress of 215 $\mathrm{MPa}$, which in turn requires a CTOD of only $0.0034 \mathrm{~mm}$. This is produced at a $\mathrm{K}$ of $24 \mathrm{MPa}$ $\mathrm{m}^{1 / 2}$, shown on both Figs. 4 and 6. Again, better dislocation density data at these lower strains would improve accuracy.

Thus, HLD predicts both $K_{\text {th1 }}$ and $K_{\text {th2 } 2}$ with good accuracy and in doing so also predicts the intersection of the $\mathrm{K}_{\text {th }}$ versus strength curves. This is unprecedented in hydrogen embrittlement studies.

Fracture surface characteristics have generally played a large role in hydrogen embrittlement studies, while they have not in this study, so some mention is in order. Based on our HLD model however, we can say that HE1 (the slow, tearing mode) should see primarily ductile features, usually called quasi-cleavage. Brittle features would be found in HE2 mode, in keeping with the literature [4], therefore HLD agrees with observed behavior. So, our model would lead us to expect much variability in fracture surfaces. It is expected that there are numerous cases where the criteria for HE1 and HE2 are both met, potentially even on the same specimen as the stress state varies across the crack front. Therefore, caution is needed when ascribing a fracture surface to a particular failure mode, as both HE1 and HE2 may coexist on a given fracture surface.

While this study concerned itself with only martensitic steels, the same factors could govern HLD in any alloy system in which the hydrogen solubility is enhanced in the FPZ, be it by enhanced defect density, hydrostatic stress effects, or the enhanced fugacity of corrosion. There is nothing in the HLD model that restricts it to certain alloy systems nor to any hydrogen source. Indeed, the similarity between martensitic steel and alloys such as A286, IN718, IN903 and 316 in Kth vs. yield strength observed recently [23], would be expected from an HLD perspective. It is expected to be equally accurate for internal hydrogen embrittlement as well as environmental hydrogen embrittlement. This will be the subject of a future study.

\section{Conclusions}

We have shown that lattice expansion by hydrogen absorption, particularly in dislocations and other traps created by deformation, is a significant and is a factor in the mechanics underlying hydrogen embrittlement.

We have used classical mechanics to demonstrate how such a lattice expansion can lead to two modes of hydrogen-induced failure in martensitic steels, the slower, HE1 tearing mode and the faster, brittle HE2 mode.

We have shown that the quantitative agreement between HLD theory and actual behavior is close in threshold stress prediction, crack velocity, and the variable behavior of different strength levels, as well as temperature.

We offer that the prediction of the transition from HE2 to HE1 especially supports the HLD model, and that the ability of the model to predict both curves of $K_{\text {th }}$ vs. yield strength and to identify their intersection is a major confirmation.

We have not evaluated any other proposed mechanisms for hydrogen embrittlement but have clarified the circumstances under which they might contribute to the overall phenomenon of hydrogen embrittlement. 
We have offered a basic mechanism that potentially unifies observations across all alloys in which hydrogen embrittlement is seen in that HLD is not alloy specific. It can exist wherever a triaxial stress state is developed during defect-creating deformation and in any environment in which hydrogen is available.

Author Contributions: Conceptualization, M.M.; methodology, M.M.; validation, M.M. and R.M.; formal analysis, M.M. and R.M.; investigation, M.M. and R.M.; resources, M.M. and R.M.; writingoriginal draft preparation, M.M.; writing - review and editing, M.M. and R.M.; visualization, M.M. and R.M.; supervision, M.M. and R.M.; project administration, M.M. and R.M. All authors have read and agreed to the published version of the manuscript.

Funding: This research received no external funding.

Data Availability Statement: The data presented in this study are available on request from the corresponding author.

Acknowledgments: The assistance of Guannan Tang, William Pingitore, and Prof. Anthony Rollett (all of Carnegie Mellon University) in sample preparation, and the donation of samples by Allegheny Technologies are gratefully appreciated.

Conflicts of Interest: The authors declare no conflict of interest. 


\section{References}

1. Troiano, A.R. The Role of Hydrogen and Other Interstitials in the Mechanical Behavior of Metals: (1959 Edward De Mille Campbell Memorial Lecture). Metallogr. Microstruct. Anal. 2016, 5, 557-569, doi:10.1007/s13632-016-0319-4.

2. Beachem, C.D. A New Model for Hydrogen-Assisted Cracking (Hydrogen "Embrittlement"). MT 1972, 3, 441-455, doi:10.1007/BF02642048.

3. Oriani, R.A. Hydrogen Embrittlement of Steels. Annu. Rev. Mater. Sci. 1978, 8, 327-357, doi:10.1146/annurev.ms.08.080178.001551.

4. Robertson, I.M.; Sofronis, P.; Nagao, A.; Martin, M.L.; Wang, S.; Gross, D.W.; Nygren, K.E. Hydrogen Embrittlement Understood. Metall and Mat Trans A 2015, 46, 2323-2341, doi:10.1007/s11661-015-2836-1.

5. Nagumo, M. Fundamentals of Hydrogen Embrittlement; Springer Singapore: Singapore, 2016; ISBN 9789811001604.

6. Comprehensive Structural Integrity; Milne, I., Ritchie, R.O., Karihaloo, B.L., Eds.; 1st ed.; Elsevier/Pergamon: Amsterdam; Boston, 2003; ISBN 9780080437491.

7. Hirth, J.P. Effects of Hydrogen on the Properties of Iron and Steel. MTA 1980, 11, 861-890, doi:10.1007/BF02654700.

8. Nibur, K.A. Measurement and Interpretation of Threshold Stress Intensity Factors for Steels in High-Pressure Hydrogen Gas; Sandia National Laboratories, Sandia Technical Report No.: SAND2010-4633, Contract No.: DE-AC04-94AL85000, 2010.

9. Pioszak, G. Hydrogen Assisted Cracking of Ultra-High Strength Steels. Ph.D. thesis, University of Virginia, 2015.

10. Bockris, J.O.; Subramanyan, P.K. A Thermodynamic Analysis of Hydrogen in Metals in the Presence of an Applied Stress Field. Acta Metallurgica 1971, 19, 1205-1208, doi:10.1016/0001-6160(71)90053-8.

11. A. R. Troiano Hydrogen Embrittlement and Stress Corrosion Cracking: A Troiano Festschrift.; American Society for Metals: Metals Park, OH, 1984.

12. Yamaguchi, T.; Nagumo, M. Simulation of Hydrogen Thermal Desorption under Reversible Trapping by Lattice Defects. ISIJ International 2003, 43, 514-519, doi:10.2355/isijinternational.43.514.

13. Hurley, D.C.; Balzar, D.; Purtscher, P.T.; Hollman, K.W. Nonlinear Ultrasonic Parameter in Quenched Martensitic Steels. Journal of Applied Physics 1998, 83, 4584-4588, doi:10.1063/1.367241.

14. Roy, A.K.; Bandyopadhyay, S.; Suresh, S.B.; Maitra, D.; Kumar, P.; Wells, D.; Ma, L. Relationship of Residual Stress to Dislocation Density in Cold-Worked Martensitic Alloy. Materials Science and Engineering: A 2006, 416, 134-138, doi:10.1016/j.msea.2005.09.092.

15. Nagumo, M. Advances in Physical Metallurgy and Processing of Steels. Function of Hydrogen in Embrittlement of HighStrength Steels. ISIJ International 2001, 41, 590-598, doi10.2355/isijinternational.41.590.

16. Nagumo, M and Takai, K. The predominant role of strain-induced vacancies in the hydrogen embrittlement of steel: Overview. Acta Mater. 2019, 165, 722-733, doi:10.1016/j.actamat.2018.12.013.

17. Tsuru T, Huang Y, Ali MR, Nishikata A. Hydrogen entry into steel during atmospheric corrosion process. Corrosion science. 2005, 47(10), 2431-40, doi:10.1016/j.corsci.2004.10.006.

18. Guo Q, Liu J, Yu M, Li S. Effect of passive film on mechanical properties of martensitic stainless steel $15-5 \mathrm{PH}$ in a neutral NaCl solution. Applied Surface Science. 2015, 327, 313-20, doi:10.1016/j.apsusc.2014.11.154.

19. Gibala, R.; Counts, W.A.; Wolverton, C. The Hydrogen Cold Work Peak in BCC Iron: Revisited, with First Principles Calculations and Implications for Hydrogen Embrittlement. Mat. Res. 2018, 21, doi:10.1590/1980-5373-mr-2017-0868.

20. Akhurst KN, Baker TJ. The threshold stress intensity for hydrogen-induced crack growth. Metallurgical Transactions A. 1981, 12(6), 1059-70, doi:10.1007/BF02643487.

21. Eshelby, J. D. The Determination of the Elastic Field of an Ellipsoidal Inclusion and Related Problems. Proc. R. Soc. Lond. A Math. Phys. Sci., 1957, 241 (1226), 376-396.

22. Kinaev, N.N.; Cousens, D.R.; Atrens, A. The crack tip strain field of AISI 4340: Part III Hydrogen Influence Journal of Materials Science 1999, 34, 4931-4936, doi:10.1023/A:1004751420033.

23. Moody, N. R., W. M. Garrison, S. L. Robinson, M. W. Perra, and W. W. Gerberich. Microstructure Effects on Hydrogen Induced Crack Growth Susceptibility of a High Strength Iron Based Superalloy. No. SAND2019-12226C. Sandia National Lab.(SNL-CA), Livermore, CA (United States), 2019. 